COPD [2]. In addition, in our recent study, increases in $P$ pa were associated with higher serum levels of high-sensitivity C-reactive protein (hsCRP), raising the possibility of a pathogenetic role of low-grade systemic inflammation in the pathogenesis of pulmonary hypertension secondary to COPD [3]. Since activation of the renin-angiotensin system is likely to contribute to inflammatory processes [4], we performed a post hoc analysis on the potential relationship between the I/D ACE gene polymorphism and circulating hsCRP levels in 72 patients with clinically stable COPD (56 male, mean \pm SD age $65.1 \pm 10.5 \mathrm{yrs,}$ forced expiratory volume in one second (FEV1) $45.8 \pm 17.4 \%$ predicted, arterial oxygen tension $\left.\left(\mathrm{Pa}_{2} \mathrm{O}_{2}\right) 8.2 \pm 1.8 \mathrm{kPa}\right)$ who were participants in our previous studies $[2,3]$. The I/D ACE polymorphism was determined as previously described [2]. Serum hsCRP levels were assessed in samples of peripheral venous blood drawn from the antecubital vein by chemiluminescent immunoassay (Randox, Crumlin, UK).

Serum hsCRP differed significantly between the II, ID, and DD groups (median (25th-75th percentile): 1.4 (0.9-2.7) versus 2.7 (1.5-6.1) versus $3.8(1.8-10.4) \mathrm{mg} \cdot \mathrm{L}^{-1}$, respectively; ANOVA on ranks, $\mathrm{p}<0.05)$. Moreover, the I/D ACE polymorphism predicted serum log hsCRP levels $(\mathrm{p}<0.05)$ independently of age, sex, FEV1 or $\mathrm{Pa}_{2} \mathrm{O}_{2}$.

In vitro studies on human vascular smooth muscle cells indicate that angiotensin II mediates a variety of proinflammatory effects through pleiotropic activation of nuclear factor- $\mathrm{\kappa} B$ transcription factors [4]. Importantly, angiotensin II receptor blockade significantly reduced serum hsCRP and tumour necrosis factor- $\alpha$ in patients with systemic hypertension [5], suggesting that the renin-angiotensin system, and probably its genetic determinants, plays an important role in vascular microinflammation. On one hand, it is well known that carriers of the D allelle of the I/D ACE gene polymorphism have higher serum and tissue ACE activities [6]. On the other hand, however, the potential relationships between the ACE genotype and inflammatory cytokines have not been analysed previously in COPD patients. Our pilot study revealed a significant relationship between the I/D ACE gene polymorphism and circulatory CRP levels in patients with stable COPD: serum hsCRP increased from the homozygous II to the heterozygous ID and then to the homozygous DD ACE genotype group, and the I/D ACE gene polymorphism predicted serum $\log$ hsCRP levels independently of age, sex, FEV1 or $\mathrm{Pa}_{\mathrm{a}} \mathrm{O}_{2}$. This finding might be meaningful, particularly in the light of recently published data indicating the clinical importance and predictive value of CRP in patients with COPD [7, 8]. High CRP levels correlate with poorer performance in the 6-min walk test [7] and, in addition, they relate to increased mortality [8].

Animal studies are needed to shed more light on the mechanisms related to the induction and/or potentiation of inflammatory processes by the renin-angiotensin system. Moreover, future clinical studies are necessary to assess the potential relationships between the genetic determinants of the renin-angiotensin system and systemic complications in chronic obstructive pulmonary disease.

\section{R. Tkacova and P. Joppa}

Dept of Respiratory Medicine, Faculty of Medicine and L. Pasteur Teaching Hospital, P.J. Safarik University, Kosice, Slovakia.

\section{SUPPORT STATEMENT}

This study was supported by operating grants VEGA 1/2305/ 05 of the Ministry of Education and 2005/5-FNLPKE-01 of the Ministry of Health, Slovakia.

\section{STATEMENT OF INTEREST}

None declared.

\section{REFERENCES}

1 Meysman M. Angiotensin II blockers in obstructive pulmonary disease: a randomised controlled trial. Eur Respir J 2006; 28: 670-671.

2 Tkacova R, Joppa P, Stancak B, Salagovic J, Misikova S, Kalina I. The link between angiotensin-converting enzyme genotype and pulmonary artery pressure in patients with COPD. Wien Klin Wochenschr 2005; 117: 210-214.

3 Joppa P, Petrasova D, Stancak B, Tkacova R. Systemic inflammation in patients with COPD and pulmonary hypertension. Chest 2006; 13: 326-333.

4 Kranzhofer R, Schmidt J, Pfeiffer CAH, Hagl S, Libby P, Kubler W. Angiotensin induces inflammatory activation of human vascular smooth muscle cells. Arterioscler Thromb Vasc Biol 1999; 19: 1623-1629.

5 Fliser D, Buchholz K, Haller H. Antiinflammatory effects of angiotensin II subtype 1 receptor blockade in hypertensive patients with microinflammation. Circulation 2004; 110: 1103-1107.

6 Danser AH, Schalekamp MA, Bax WA, et al. Angiotensinconverting enzyme in the human heart. Effect of deletion/ insertion polymorphism. Circulation 1995; 15: 1387-1388.

7 de Torres JP, Cordoba-Lanus E, López-Aguilar C, et al. Creactive protein levels and clinically important predictive outcomes in stable COPD patients. Eur Respir J 2006; 27: 902-907.

8 Man SF, Connett JE, Anthonisen NR, Wise RA, Taskhin DP, Sin DD. C-reactive protein and mortality in mild to moderate obstructive pulmonary disease. Thorax 2006; 61: 849-853.

DOI: $10.1183 / 09031936.00147506$

\title{
Diffuse panbronchiolitis: East meets West
}

\section{To the Editors:}

In the paper by POLETTI et al. [1] on diffuse panbronchiolitis, the authors describe the diagnostic criteria for this condition and emphasise its predominance in East Asia, having associations with human leukocyte antigen (HLA)-Bw54 and HLA-A11.

We would like to highlight the difficulties and pitfalls in reaching this rare diagnosis in a European cohort of patients by 
describing the unusual presentation of this condition in a Caucasian female, the first case described in Ireland.

A 48-yr-old female attended outpatient clinic for her fifth annual asthma review. She had experienced four infective exacerbations in the previous 12 months and reported recent large-volume sputum production, associated with worsening dyspnoea on exertion. Computed tomography (CT) thorax scan revealed bronchiectasis. The patient was treated over the subsequent 3 yrs for exacerbations of asthma and bronchiectasis.

Over this period, her pulmonary function tests began to show severe small airways obstruction. The patient was now experiencing severe dyspnoea on minimal exertion and was becoming unresponsive to inhaled bronchodilators and highdose oral steroids. Pulmonary function testing at this point revealed airflow obstruction, with a reduced forced expiratory volume in one second (FEV1) of $1.67 \mathrm{~L} \cdot \mathrm{min}^{-1}$ (66\% predicted), a forced vital capacity (FVC) of $2.31 \mathrm{~L} \cdot \mathrm{min}^{-1}$ (78\% pred) and a diffusing capacity of the lung for carbon monoxide that was $49 \%$ pred. Bronchiolitis/bronchiolectasis was now suspected. Further CT scanning demonstrated more extensive changes of bronchiectasis, associated with fine nodular opacities in both lung bases. In contrast to the usual pattern, this had a more distal distribution. The combination of these CT findings and small airways obstruction without large airway involvement on pulmonary function testing prompted referral for thoracoscopic lung biopsy. Exhaustive analysis of the tissue, including a second opinion from an international centre, revealed a histological diagnosis of diffuse panbronchiolitis. The patient was commenced on erythromycin $500 \mathrm{mg}$ b.i.d. with a dramatic response. Macrolide treatment was continued over the next 2 yrs with a significant reduction in sputum production, improvement in spirometry (FEV1 $2.35 \mathrm{~L} \cdot \mathrm{min}^{-1}$ and FVC $\left.3.9 \mathrm{~L} \cdot \mathrm{min}^{-1}\right)$, resolution of wheeze and improved exercise tolerance comparable to that of $10 \mathrm{yrs}$ earlier. Over the next few years, attempts were made to reduce erythromycin therapy with little success. The patient's debilitating symptoms always returned. She remained on erythromycin $500 \mathrm{mg}$ b.i.d. maintenance dose and managed to complete a 200-mile sponsored walk.

This case highlights the importance of considering this diagnosis or certainly the forme fruste mentioned by POLETTI et al. [1] in their review, in the group of patients with asthma and/or bronchiectasis who show no improvement with conventional therapies, including high-dose oral steroid, and who actually decline rapidly despite treatment. Something as simple as erythromycin could be the difference between severe morbidity or death and walking 200 miles!

\section{E.E. McGrath, A.M. McLaughlin and M.X. FitzGerald \\ St. Vincent's University Hospital Dublin, Dublin, Ireland.}

\section{STATEMENT OF INTEREST}

None declared.

\section{REFERENCES}

1 Poletti V, Casoni G, Chilosi M, Zompatori M. Diffuse panbronchiolitis. Eur Respir J 2006; 28: 862-871.

DOI: $10.1183 / 09031936.00157606$

\section{Exposure to second-hand smoke: a population-based survey in Spain}

\section{To the Editors:}

In 1972, a US Surgeon General's report, "The Health Consequences of Smoking", addressed the topic of passive smoking [1]. More than three decades later, another Surgeon General's report, "The Health Consequences of Involuntary Exposure to Tobacco Smoke", concluded that a causal relationship exists between second-hand smoke (SHS) exposure experienced by lifelong nonsmokers and several diseases, including lung cancer and coronary heart disease [2].

Despite the report's findings, legislation promoting population protection against this ambient carcinogen [3] has been slow to take hold globally. The exposure of the Spanish population remains unknown and existing data on prevalence of exposure are limited to selected areas or specific populations [4, 5]. An anti-smoking law was recently implemented in Spain [6], with the aim of combating this health problem by decreasing population exposure to SHS in employment and public settings.

Between December 2004 and January 2005, we developed a study on tobacco use and SHS exposure among the general population in the northern Spanish region of Galicia. The source of sampling was the regional healthcare card system, which covers $97 \%$ of the population. The study yielded SHS exposure data collected in Galicia, based on a representative population sample. Galicia is located in the north-west of Spain and was estimated to have a population of 2.7 million in 2004, with a smoking prevalence of $29 \%$ (95\% confidence interval $27.8-30.2 \%$ ). People who smoked daily or occasionally were classified as smokers.

One of the study's objectives was to estimate exposure to SHS in the general population, aged 16-74 yrs. The sampling unit was the individual and the sample $(n=6,492)$ was representative by sex and was divided into age groups: 16-24, 25-44, and 45-74 yrs. Data collection was carried out primarily through telephone interviews (90\%); however, $10 \%$ of the interviews were conducted in person, in order to avoid under-representation of people without phone facilities.

The Galician Smoking Interview Survey included several questions that aimed to estimate SHS exposure within the three microenvironments most commonly frequented by the 